\title{
Comparison of Scapular Muscles Activation with Shoulder Retraction and Shoulder Elevations in Individuals with Scapular Dyskinesia
}

\author{
P. Gautami Easwaran ${ }^{1}$, Dr. Urvashi Sharma², Dr. Tushar J. Palekar ${ }^{3}$ \\ IIntern, Dr. D Y Patil College of Physiotherapy, Pune, Maharashtra, India \\ ${ }^{2}$ Assistant Professor, Dr. D Y Patil College of Physiotherapy, Pune, Maharashtra, India \\ ${ }^{3}$ Professor and Principal, Dr. D Y Patil College of Physiotherapy, Pune, Maharashtra, India
}

\begin{abstract} proposed that abnormal scapular kinematics may be linked to weakness of scapular muscle. resulting in increased strength, stability, range of motion, and reduced muscle and joint pain. the interscapular distance in indivudals with scapular dyskinesia.

\section{Objectives:}

1. To assess Interscapular distance post shoulder retraction exercise.

2. To assess Interscapular distance post shoulder shrugging exercise.

3. To assess Interscapular distance post shoulder retraction + shrugging exercise.

4. To compare the effectiveness of these exercises on scapular dyskinesia.
\end{abstract}

Background : Scapular dyskinesis is a condition that is commonly associated with shoulder pathology but is also present in asymptomatic individuals, and it is believed to be a risk factor for further injury. Evidence suggests that patients with rotator cuff-related shoulder pain present scapular kinematic abnormalities such as decreased scapular upward rotation, decreased scapular posterior tipping, and external rotation. It has been

Muscle Activation is a very specific, non-medical, mechanically based process of evaluating and treating muscular imbalance and dysfunction. Its main goal is to increase the contractile capability of your muscles,

The study was conducted to determine whether shoulder elevations and retractions showed any difference in

Materials and Methodology : An intervention based study comprising of 30 female individuals. The subjects were randomly divided into 3 groups, Group A was given combined exercises of shoulder retraction and elevations. Group B was given shoulder retraction and Group C was given shoulder elevations.The samples were assessed pre and post with the help of Interscapular distance.

Results : Shoulder retractions when given along with shoulder elevations was clinically significant pre and post . Likewise, Shoulder retractions and elevations when given separately were also clinically significant pre and post. The comparison of all the three groups showed that all were equally effective in treating scapular dyskinesia.

Conclusion : The study concludes that all three forms of exercise showed similar results in reducing the Inter scapular distance in individuals with scapular dyskinesia.

Keywords : Inter Scapular Distance, Shoulder Retraction, Shoulder Elevation, Scapular Dyskinesia. 


\section{INTRODUCTION}

Scapular dyskinesia is an alteration in normal scapular position and motion. It is characterized by prominence of the scapular medial border and/or inferior angle relative to the thoracic cage in the static position or in dynamic motion; early scapula elevation or shrugging during arm elevation; or inadequate upward and downward rotation of the scapula during arm elevation or lowering .

Muscle Activation is a very specific, non-medical, mechanically based process of evaluating and treating muscular imbalance and dysfunction. Its main goal is to increase the contractile capability of your muscles, resulting in increased strength, stability, range of motion, and reduced muscle and joint pain. Muscle Activation Technique is known to be an effective treatment for injury recovery and rehabilitation, as well as chronic pain management for a variety of neuromuscular disorders.

The shoulder complex is made up of several joints. The glenohumeral joint (GHJ) and the scapulothoracic joint (STJ) are thought to be important in the shoulder complex and make up a large portion of shoulder movement. There are numerous studies on scapulo-humeral rhythm and muscle activity, especially during shoulder elevation. Therefore, although there is a lot of information on shoulder elevation, there is less information on horizontal abduction and adduction and internal and external rotation of the shoulder joint. Shoulder movement during activities of daily living and sport is diverse and does not just comprise shoulder elevation. Therefore, an investigation of shoulder movements other than elevation will further the understanding of shoulder performance in activities of daily living. ${ }^{1}$

The scapula supports the humerus, and scapular stability is important for shoulder movement. Scapular muscle activity and scapular movement are different in patients with shoulder disease compared to healthy subjects. For example, scapular kinematic alterations associated with shoulder impingement, rotator cuff tendinopathy, rotator cuff tears, adhesive capsulitis, and so on, and these patients often reduced serratus anterior and increased upper trapezius activation. And it was reported that the combination of standard rehabilitation interventions and scapulothoracic joint control training exercises were an effective treatment of the shoulder joint dysfunction. Therefore, research on exercises to change scapular muscle activity has led to the recognition of the importance of scapular stability for shoulder movement in normal scapular muscle activity. However, apart from shoulder elevation, the mechanism of scapular stability, especially in isometric contraction and movement of the shoulder joint, remains unknown. Ijiri et al. previously investigated scapular muscle activity during isometric contraction of the shoulder joint during internal and external rotation, and revealed differences in activity among the scapular muscles. This difference is based on the constant rule that muscles with same movement as the scapular movement have higher activity. In addition, the reaction time against external force is also different for each scapular muscle. In the previous study, the muscles with higher activity reacted earlier compared to the other scapular muscles, with similar results for the agonist muscles of GHJ. We considered that the scapular muscles are divided into agonist and the antagonist muscles and that the agonist muscles of STJ have the 
same function against external force as the agonist muscles of GHJ and contribute to scapular stability. ${ }^{1}$

The scapular muscles work synergistically to control the scapular movement, and patterned scap-ular muscle activations are necessary for optimal scapular stabilization. The upper trapezius (UT), lower trapezius (LT) and serratus anterior (SA) are working together to move the scapula into the upward rotation. The middle trapezius (MT) and rhomboid muscles retract the scapula and Lower trapezius cause upward rotation and depression of the scapula. In addition, during arm elevation, LT contributes to posterior tilt and external rotation of the scapula ${ }^{2}$

The scapular retraction exercises are widely used by clinicians in an aim to balance the activity of the scapular muscles as well as the rotator cuff muscles in different upper-limb positions to improve the scapular kinematics .

The general movement pattern of the scapula observed during scapular retraction exercises was the upward rotation, external rotation and posterior tilt, and in theory, this pattern results in increasing the acromio-humeral distance. However, there is limited information that shows how trapezius muscle activity changes during scapula retraction exercise with different shoulder abduction angles. Understanding changes in the trapezius muscle activity among retraction exercises may help guide the clinicians to select activities that are most appropriate for the rehabilitation program. ${ }^{2}$

\section{METHODS AND MATERIAL}

Study Location: Study was conducted in Dr D Y Patil College of Physiotherapy, Pune.
Sampling Method: A random selection was done among 60 samples. 30 subjects meeting the inclusion and exlusion criteria were recruited. They were selected based on a postural analysis done which included if the individuals presented with protracted shoulders or any neck pain

Procedure and data collection: Subjects were randomly divided into 3 groups; Group A, Group B, Group C. Group A subjects $(\mathrm{n}=10)$ were taught combined movements of shoulder retraction and elevation, subjects in Group B $(n=10)$ were taught Shoulder retraction, subjects in Group C ( $n=10)$ were taught shoulder elevations.

Group A: On day 1 Therapist demonstrated shoulder shrugging with retraction together to the subjects and asked them to repeat the exercises for 3 repititons after the therapist said "Elevate your shoulders towards your ears and along with that squeeze your shoulder blades back", they were asked to hold the position for 20 seconds and take rest for $15 \mathrm{~s}$ before starting the next exercise. Subjects were guided to breathe normally throughout the exercise. Followed by this trial, 5 minutes of break was given , Individuals then performed 20 repetitions of the shoulder shrugs with $10 \mathrm{~s}$ hold. This was continued for days 2, 3, 4, 5,6.

Group B: On day 1 Therapist demonstrated the Shoulder retraction isometric exercises to the subjects and asked them to repeat the exercises for 3 repetitions after the therapist says "squeeze your shoulder blades back", they were asked to hold the position for 20 seconds and take rest for $15 \mathrm{~s}$ before starting the next exercise. Subjects were guided to breathe normally throughout the exercise (not to hold their breath to avoid Valsalva maneuver) Followed by this trial, 5 minutes of break was given, individuals were then asked to perform 20 repetitions 
of the Shoulder Retraction exercises with $10 \mathrm{~s}$ hold.

This was then continued for days 2,3,4,5,6.

Group C : On day 1 Therapist demonstrated shoulder shrugging exercise to the subjects and asked them to repeat the exercise for 3 repetitions after the therapist said "Elevate your shoulder and bring them towards your ears", they were asked to hold the position for 20 seconds and then take rest for $15 \mathrm{~s}$ before starting the next exercise. Subjects were guided to breathe normally throughout the exercise. Followed by this trial, 5 minutes of break was be given, Individuals were asked to perform 20 repetitions of the shoulder shrugs with $10 \mathrm{~s}$ hold. This will be continued for days $2,3,4,5,6$.

Outcome measure i.e Interscapular distance was recorded in the data collection form (Appendix 3, 4) on $1^{\text {st }}$ day of treatment and was recorded again on day 7 post treatment.

\section{RESULTS AND DISCUSSION}

In this study, 30 individuals having scapular dyskinesia were selected and divided into group $\mathrm{A}(\mathrm{n}=10)$, Group $\mathrm{B}(\mathrm{n}=10)$ and group $\mathrm{C}(\mathrm{n}=10)$ randomly and Interscapular Distance was compared before and after the intervention and graphically represented.

TABLE 1. GROUP A ISD PRE POST

\begin{tabular}{|l|l|l|l|}
\hline GROUP & MEAN & P VALUE & INFERENCE \\
\cline { 1 - 2 } PRE & $1.95 \pm 0.4378$ & 0.000 & SIGNIFICANT \\
\cline { 1 - 2 } POST & $1.05 \pm 0.4378$ & & \\
\hline
\end{tabular}

\section{GRAPH NO.1}

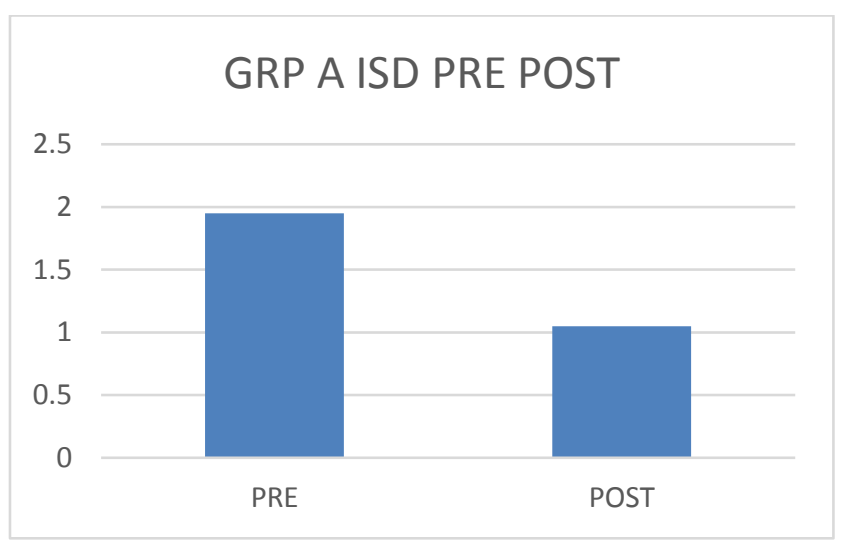

\section{INTERPRETATION}

Table 1 and Graph 1 shows within group analysis of group A ISD ups pre and post which includes how many push ups the individual was able to do pre and post . Paired t-test was applied and the result shows statistically significant change pre and post intervention as $\mathrm{p}<0.005$. This shows that group $\mathrm{A}$ treatment was effective in strengthening in mild degrees in individuals with scapular dyskinesia

TABLE 2. GROUP B ISD PRE POST

\begin{tabular}{|l|l|l|l|}
\hline GROUP & MEAN & $\begin{array}{l}\text { P } \\
\text { VALUE }\end{array}$ & INFERENCE \\
\hline PRE & $1.88 \pm 0.4264$ & 0.000 & SIGNIFACANT \\
\hline POST & $1.24 \pm 0.4926$ & & \\
\hline
\end{tabular}




\section{GRAPH NO.2}

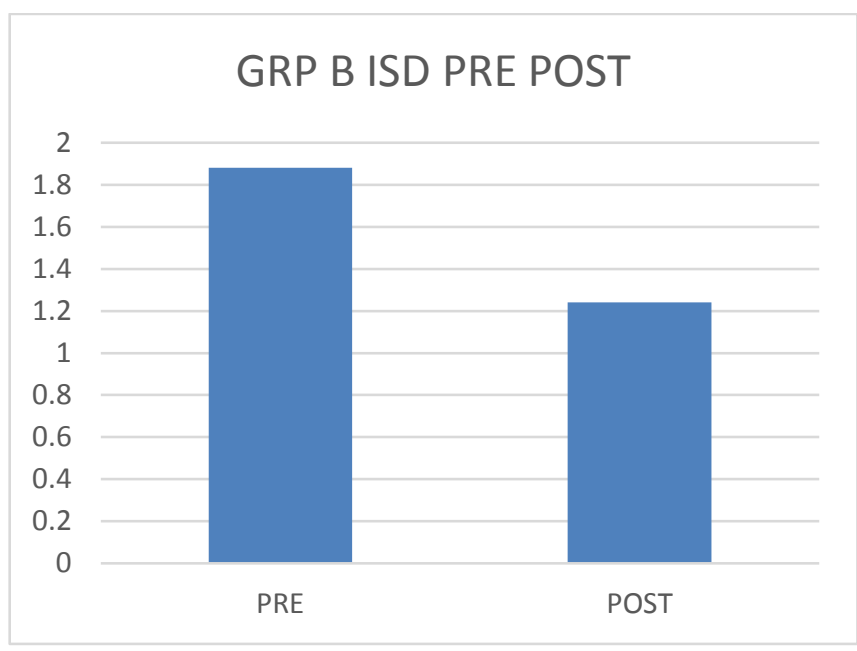

\section{INTERPRETATION:}

Table 2 and Graph 2 shows within group analysis of group B ISD ups pre and post which includes how many push ups the individual was able to do pre and post . Paired t-test was applied and the result shows statistically significant change pre and post intervention as $p<0.005$. This shows that group $B$ treatment was effective in strengthening in mild degrees in individuals with scapular dyskinesia.

TABLE 3. GROUP C ISD PRE POST

\begin{tabular}{|l|l|l|l|}
\hline GROUP & MEAN & $\begin{array}{l}\text { P } \\
\text { VALUE }\end{array}$ & INFERENCE \\
\cline { 1 - 2 } PRE & $1.89 \pm 0.3814$ & 0.000 & SIGNIFICANT \\
\cline { 1 - 2 } POST & $1.28 \pm 0.4367$ & & \\
\hline
\end{tabular}

\section{GRAPH NO.3}

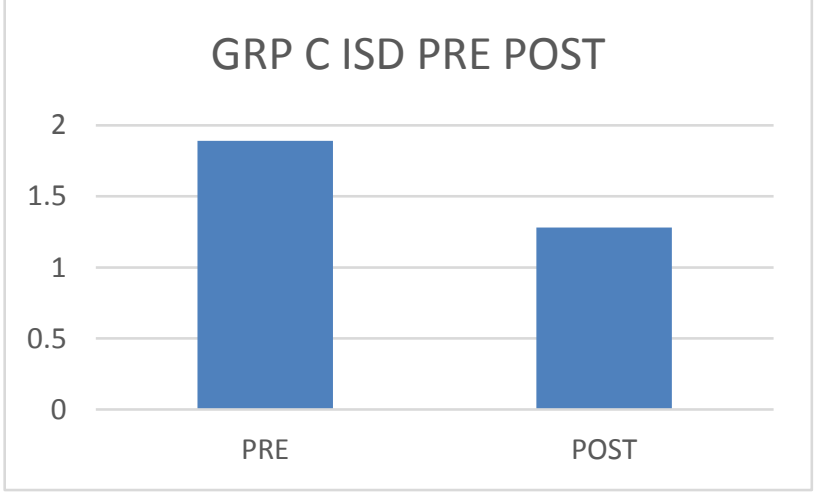

\section{INTERPRETATION:}

Table 3 and Graph 3 shows within group analysis of group C ISD ups pre and post which includes how many push ups the individual was able to do pre and post . Paired t-test was applied and the result shows statistically significant change pre and post intervention as $p<0.000$. This shows that group $C$ treatment was effective in strengthening in mild degrees in individuals with scapular dyskinesia

\section{DISCUSSION}

The present study on "Comparison of scapular muscles activation with shoulder retraction and shoulder elevations in individuals with scapular dyskinesia" was conducted to determine whether scapular retractions and elevations intervention had any effects on individuals with scapular dyskinesia residing in Pimpri, Pune city.The study was conducted with 60 samples by simple random sampling, out of which 30 were selected having scapular dyskinesia.

The objective of this study was to determine whether performing shoulder retraction and elevations had any effects in reducing the interscapular distance in individuals with scapular dyskinesia.

Our study says that dyskinesia was also seen in individuals between the age group of 18-30 because this age group comes under the working and college going population, wherein they tend to adapt a single posture for a prolonged period of time.

In Group A, the results showed that the interscapular distance had reduced significantly $(\mathrm{p}<0.000)$ before and after treatment.

In Group B, the results showed that the interscapular distance had reduced significantly $(\mathrm{p}<0.000)$ before and after treatment. 
In Group C, the results showed that the interscapular distance had reduced significantly $(\mathrm{p}<0.000)$ before and after treatment.

When the comparison of all the groups were performed, all three groups showed similar amount of difference in the interscapular distance. They showed no significant difference in the data recorded. $(\mathrm{p}<0.005)$.

Russ Paine studied that an effective exercise program for rehabilitation should include improving the strength and function of the muscles that control the position of the scapula. He also mentioned that implementation of the scapular program can begin early in the rehabilitation protocol and progress to more aggressive strengthening approaches. Scapular strengthening should be included as a part of all rehabilitation programs relating to the shoulder complex. Advancement in the knowledge of shoulder complex biomechanics and related EMG patterns has allowed for the development of strengthening exercises that maximally address these "anchor" muscles. As there are techniques evolving to objectively measure scapular position and dynamic function, the interaction between the scapula and glenohumeral joint can be further clarified.

It can be further concluded that these interventions could be given individually or in combination with each other as it may suit the person.

\section{v. CONCLUSION}

The study concludes that both shoulder retraction and shoulder elevation helped in reduction of interscapular distance in individuals with scapular dyskinesia.

\section{REFERENCES}

[1]. Ijiri $\mathrm{T}$, Takagi $\mathrm{R}$ and Suzuki $\mathrm{T}$ Isometric Contraction of Scapular Muscles Activities during horizontal Abduction and Adduction of the shoulder (2017) J.Nov Physiother ,Vol 2, Issue 7.

[2]. Harput G, Guney -Deniz H, Duzgun I Upper to Middle Trapezius Activation Ratio during Scapular Retraction Exercise at Different Shoulder Abduction Angles .(2017) Turk J PhysiotherRehab , 28(3):111-117

[3]. Escamilla RF, Yamashiro K, Paulos L, Andrews JR Shoulder Muscle Activity and Function in common shoulder rehabilitation exercises (2009) Sports Med, 39(8):663-85

[4]. Castelein B , Cools A, Parlevliet T, Cagnie B (2016) Modyfying the shoulder joint position during shrugging and retraction exercises alters the activation of the medial scapular muscles, Manual Therapy ,21: 250-5

[5]. Wickham J, Pizzari T, Stansfeld K, Burnside A, Watson L Quantifying 'normal' shoulder muscle activity during abduction (2009) Journal of Electromyography and Kinesiology ,2010 Apr, (20)2:212-22

[6]. McCann PD, Wootten ME, Kadaba MP, Bigliani LU A kinematic and Electromyographic study of shoulder rehabilitation exercises Clin Orthop Relat Res Mar; (288): 179-88

[7]. Decker M, Hintermeister R, Faber K, Hawkins $\mathrm{R}$ Serratus Anterior Muscle Activity during selected rehabilitation exercises. The American Journal of sports medicine, vol 27, No 6:784

[8]. Curtis T, Roush J The Lateral Scapular Slide Test: A reliability study of males with and without shoulder pathology .North American Journal of Sports Physiotherapy, 2006 August, Vol 1, No.3 
[9]. Kendall FP, McCreary EK, Provance PG, Rodgers MM \& Romani WA Muscles Testing and Function with posture and pain.

[10]. Deng S, Chen K, Ma Y The Influence of Test Positions on Clinical Assesment for Scapular Dyskinesis.

[11]. Nodehi Moghadam, Afsun et al. "The Effectiveness of Exercise Therapy on Scapular Position and Motion in Individuals With Scapular Dyskinesis: Systematic Review Protocol" JMIR research protocols vol. 6,12 e240. 13 Dec. 2017, doi:10.2196/resprot.8011

\section{Cite this article as :}

P. Gautami Easwaran, Dr. Urvashi Sharma, Dr. Tushar J. Palekar, "Comparison of Scapular Muscles Activation with Shoulder Retraction and Shoulder Elevations in Individuals with Scapular Dyskinesia", International Journal of Scientific Research in Science and Technology (IJSRST), Online ISSN : 2395-602X, Print ISSN : 2395-6011, Volume 6 Issue 2, pp. 658-664, March-April 2019. Available at doi : https://doi.org/10.32628/IJSRST1962115 Journal URL : http://ijsrst.com/IJSRST1962115 